\title{
Ectopic expression of activation-induced cytidine deaminase caused by epigenetics modification
}

\author{
MASAYUKI TATEMICHI, HARUMI HATA and TOSHIO NAKADATE \\ Department of Hygiene and Preventive Medicine, Showa University, School of Medicine, \\ 1-5-8 Hatanodai, Shinagawa-ku, Tokyo 142-8555, Japan
}

Received June 8, 2010; Accepted August 27, 2010

DOI: 10.3892/or_00001055

\begin{abstract}
Recently studies have shown that ectopic expression of activation-induced cytidine deaminase (AID) plays an important role in carcinognesis and cancer progression of inflammatory-associated cancers. Here, we examined the molecular mechanism of ectopic expression of AID in cancer cells, and whether or not nitric oxide (NO) modulates this expression, as NO is known to cause chemical deamination of the cytidine. In several cancer cell lines, treatment with the DNA methyltransferase (Dnmt) inhibitor 5-Aza-dC effected expression of AID by TNF- $\alpha$, and expression was further induced by additional treatment with histone deacetylase (HDAC) inhibitors with no stimulation. The CpG sites located in the promoter and exon 1 region of the AID gene in cancer cells were found to be hypomethylated in correlation with AID expression levels. Further, administration of HDAC inhibitors also induced expression of inducible nitric oxide synthase (iNOS) in cancer cells treated with 5-Aza-dC. Interestingly, administration of S-nitroso-L-glutathione (GSNO) a nitric oxide (NO) donor, was found to enhance AID and iNOS expression in LoVo cells treated with 5-Aza-dC. Our findings suggest that AID and iNOS expression in cancer cells may be modified by epigenetic mechanisms, and that NO may further enhance AID and iNOS expression. Given recent plans to introduce Dnmt and HDAC inhibitors as novel cancer treatments, these findings regarding the potential for Dnmt and HDAC inhibitors to enhance expression of AID and iNOS, resulting in further cancer progression, might be taken into consideration.
\end{abstract}

\section{Introduction}

Activation-induced cytidine deaminase (AID) initiates somatic hypermutation, class-switch recombination and gene

Correspondence to: Dr Masayuki Tatemichi, Department of Hygiene and Preventive Medicine, Showa University School of Medicine, 1-5-8 Hatanodai, Shinagawa-ku, Tokyo 142-8555, Japan E-mail: tatemichi@med.showa-u.ac.jp

Key words: activation-induced cytidine deaminase, inducible nitric oxide synthase, methylation, histone deacetylase inhibitor conversion in immunoglobulin genes (1), and expression in activated $B$ cells in the germinal center of lymphoid tissue is strictly regulated (2). However, recent studies have shown that pathological ectopic AID expression in cancer cells and epithelial cells can lead to development of inflammationassociated cancers (3-5). Further, ectopic expression of AID can increase the mutation rate in genes such as the p53 gene (6).

Inducible nitric oxide synthase (iNOS) is responsible for producing a large amount of nitric oxide (NO). Pathophysiologically, iNOS is induced by inflammatory cytokines and lipopolysaccharides (7). Several reports have noted iNOS expression in several types of inflammation-associated cancers, and its expression is associated with p53 gene mutation and an overall poor prognosis (8). NO has been shown to have potentially pro-tumor effects, such as antiapoptotic effects, induction of production of vascular endothelial growth factor and matrix metalloproteinases and suppression of immunoreactions (9-14). Further, NO is known to cause chemical deamination at the cytidine in AID (15).

Methylation of $\mathrm{CpG}$ sites in the promoter region and modification of histone proteins is known to suppress gene expression, and hypermethylation of such $\mathrm{CpG}$ sites in tumor suppressor genes plays a critical role in carcinogenesis (16). In light of these previous findings, DNA methyltransferase (Dnmt) and HDAC inhibitors have been suggested as novel cancer treatments (17). However, we hypothesize that ectopic expression of genes having harmful effects, such as AID, may be caused by deregulation due to epigenetics-based genes silencing, including demethylation in DNA and acetylation in histone proteins. Here, to prove our hypothesis, we examined whether or not deregulation of epigenetics is involved in the ectopic expression of AID, and whether or not NO modulates this expression.

\section{Materials and methods}

Cell culture and protocol. Cancer cells (PANC-1, AsPC-1, BxPC-3, DLD-1, SW480, LoVo, MNK45, MNK28 and MCF-7) were cultured at $37^{\circ} \mathrm{C}$ and $5 \% \mathrm{CO}_{2}$ in Dulbecco's modified Eagle's medium (Life Technologies Japan, Ltd., Tokyo, Japan) containing $4.5 \mathrm{~g} / 1$ glucose (Life Technologies Japan, Ltd.) and supplemented with $10 \%$ fetal bovine serum, $2 \mathrm{mM}$ l-glutamine, $0.1 \mathrm{mM}$ non-essential amino acids and an antibiotic mix (Life Technologies Japan, Ltd.). HCT-116 cells were cultured in McCoy's 5A medium. 
A)

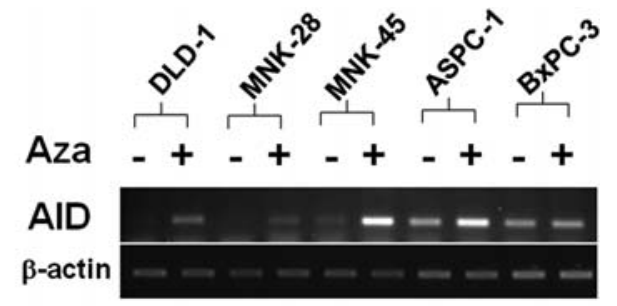

B)

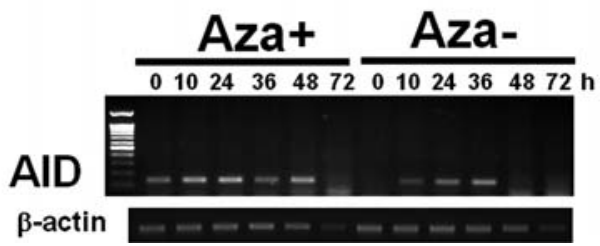

C)

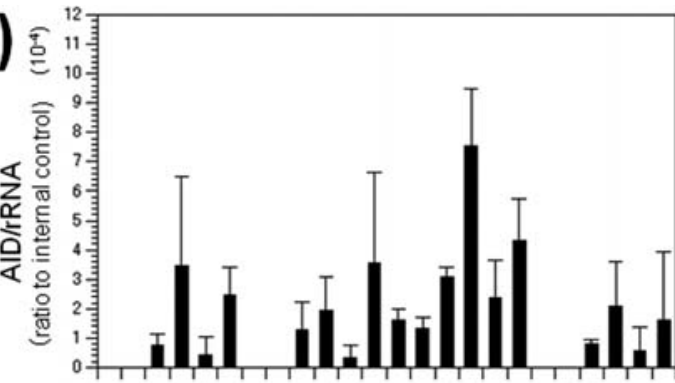

D)
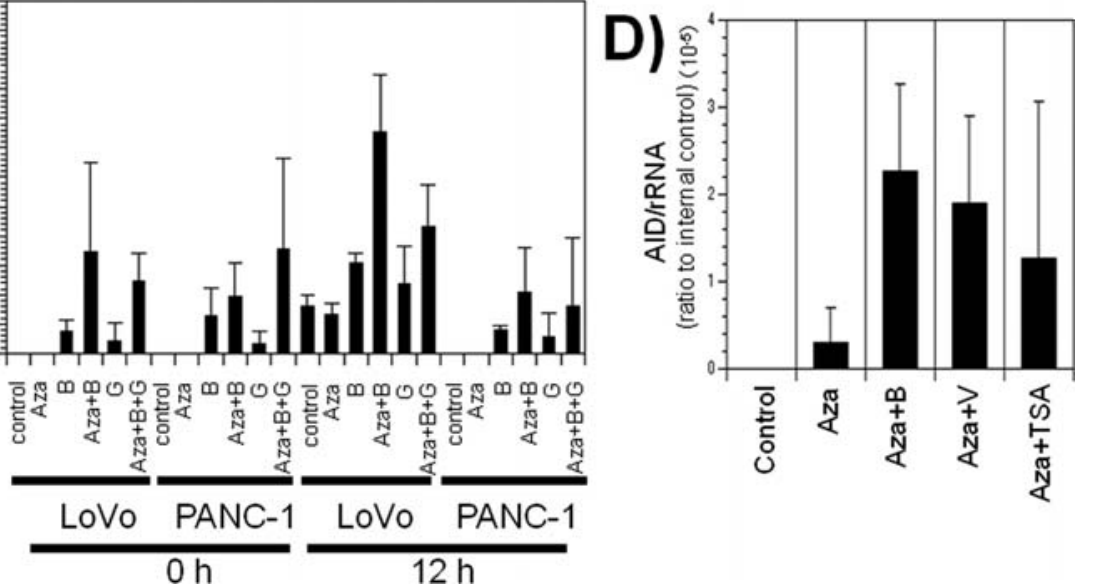

Figure 1. Effects of the treatment with Dnmt and HDAC inhibitors on AID expression. (A) Human cancer cell lines (DLD-1, MNK-28, MNK-45, AsPC-1 and BxPC-3) were treated with (+) or without (-) the Dnmt inhibitor 5-Aza-dC (5 $\mu \mathrm{M})$ for three days, and AID expression was examined by RT-PCR at $10 \mathrm{~h}$ after stimulation with TNF- $\alpha(50 \mathrm{ng} / \mathrm{ml})$. (B) Effects of 5-Aza-dC on time-course AID expression after stimulation with TNF- $\alpha$ (50 ng/ml) were examined using RT-PCR in PANC-1 cells. (C) Effects of treatment with 5-Aza-dC + HDAC inhibitor (sodium butyrate) or S-nitroso-L-glutathione (GSNO), a NO donor, on AID expression by real-time PCR. Cells were treated with 5-Aza-dC $(5 \mu \mathrm{M})$ for three days and then butyrate $(2 \mathrm{mM})$ or GSNO (0.5 mM) were added to the medium for $24 \mathrm{~h}(0 \mathrm{~h})$. Sequentially cells were stimulated with TNF- $\alpha(50 \mathrm{ng} / \mathrm{ml})$ for $12 \mathrm{~h}$ and AID mRNA was determined by real-time PCR. (D) Effects of treatments with HDAC inhibitors [namely sodium butyrate $(2 \mathrm{mM})$, valproic acid $(5 \mathrm{mM})$ and Trichostatin A (TSA; $0.1 \mu \mathrm{M})$ ] for $24 \mathrm{~h}$ after a 3 -day treatment with 5-Aza-dC on AID expression were analyzed by real-time PCR in LoVo cells. To quantify the expression level of AID, PCR products of AID and rRNA were cloned into pTAC-1 vector, and copy numbers per microlitre were determined by standard for absolute quantification. Aza, 5-Aza-dC; B, sodium butyrate; G, GSNO; V, valproic acid; TSA, Trichostatin A.

Cells were treated with $5 \mu \mathrm{M}$ of 5-Aza-dC (Sigma-Aldrich, St. Louis, MO), a Dnmt inhibitor, for three days, after which the cells were treated with either one of several types of HDAC inhibitor: sodium butyrate (Sigma-Aldrich), valproic acid (Sigma-Aldrich) or Trichostatin A (TSA) (Wako Pure Chemical Industries, Ltd., Osaka, Japan); or with one of several NO donors: S-nitroso-L-glutathione (GSNO) and S-nitorosoN-acetyl-D,L-penicillamine (SNAP; Cayman Chemical Co., Ann Arbor, MI, USA) with or without TNF- $\alpha$ (50 ng/ml; Wako Pure Chemical Industries, Ltd.).

Reverse transcription-polymerase chain reaction ( $R T-P C R)$ and real-time $P C R$. Cells were harvested, and total RNA was isolated using a FastPure RNA kit (Takara Bio Inc., Shiga, Japan). First-strand cDNA was synthesized using a commercial kit (ReverTra Ace qPCR RT kit; Toyobo Co., Ltd., Osaka, Japan) in a reaction volume of $10 \mu \mathrm{l}$ containing $500 \mathrm{ng}$ of total RNA, according to the manufacturer's instructions.
To detect the appropriate genes, first-strand cDNA was amplified by PCR using sets of oligonucleotide primers. RTPCR and quanti-tative real-time RT-PCR for human AID amplification was performed as described elsewhere (4). The primers used in the present study for RT-PCR and quantitative real-time PCR for iNOS and $\beta$-actin were as follows: iNOS-F-5'-CCGCTTCGATGTGGTCCCCCTGGTCCTG-3' iNOS-R-5'-TCTCTTCTCTTGGGTCTCCGCTTCTCGTC-3', actin-F-ATGATATCGCCGCGCTCGTCGTC and actin-R-CC AGAGGCGTACAGGGATAGCAC for RT-PCR, and iNOSF-5'-GAGGTGGCCATGGAACATCC-3', iNOS-R-5'-CCC ATTCTCCTGCCCACTTC-3', rRNA-F-5'-AAACGGCTA CCACATCCAAG-3' and rRNA-R-5'-CAATTACAGGGC CTCGAAAG-3' for real-time PCR. Real-time PCR was conducted using the ABI-PRISM 7900HT Sequence Detection System (Applied Biosystems, Foster, CA, USA) and power SYBR-Green (Applied Biosystems) following the protocol provided by the manufacturer. To quantify the 


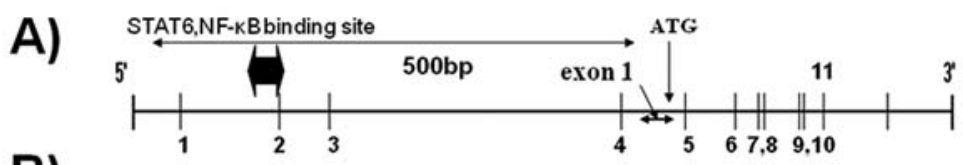

B)

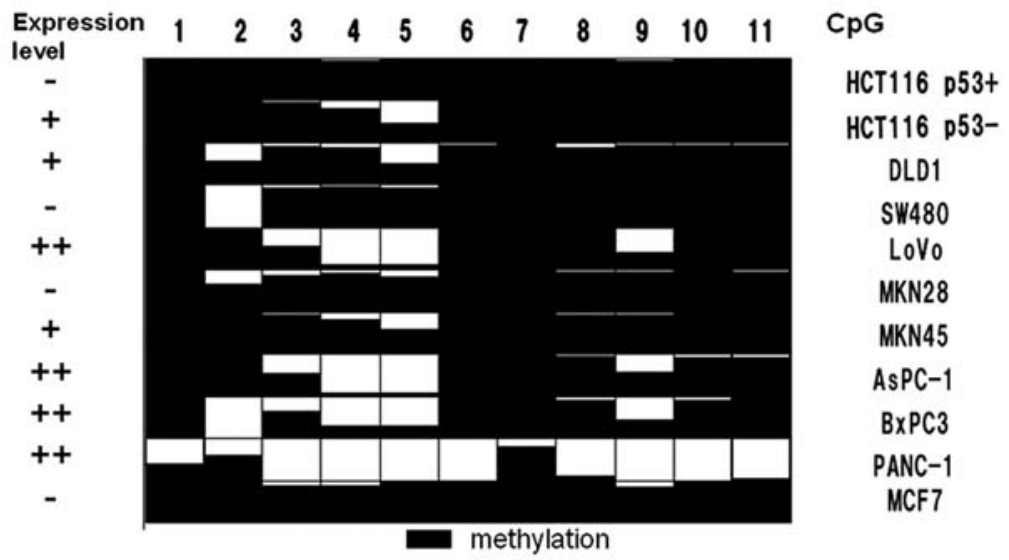

Figure 2. Methylation state at CpG sites in the promoter and exon 1 regions of the AID gene. (A) Eleven methylated CpG sites in the AID promoter and exon 1 regions were determined by the bisulfite-sequence method. (B) Methylation state in genomes extracted from human cancer cell lines was determined using the bisulfite-squence method. AID mRNA expression at $10 \mathrm{~h}$ after TNF- $\alpha(50 \mathrm{ng} / \mathrm{ml})$ expression was quantified by image analysis and classified into one of three categories: -, + or ++

expression level of AID mRNA, PCR products of AID and rRNA were cloned into pTAC-1 vector (BioDynamics Laboratory Inc., Tokyo, Japan), and copy numbers per microlitre were determined by standard for absolute quantification.

Western blotting. Total protein homogenates prepared from cultured cells using radioimmunoprecipitation assay buffer (25 mM Tris/HCl, pH 7.6, $150 \mathrm{mM} \mathrm{NaCl}, 1 \% \mathrm{NP}-40,1 \%$ sodium deoxycholate, $0.1 \%$ SDS; Thermo Fisher Scientific K.K., Kanagawa, Japan) containing complete mini and PhosSTOP Proteins (50 $\mu \mathrm{g}$; Roche Diagnostics, Mannheim, Germany) were separated on an $8 \%$ sodium dodecyl sulfate polyacrylamide gel and electrotransferred to a nitrocellulose membrane (Hybond-ECL; GE Healthcare UK, Ltd., Buckinghamshire, UK). Blots were blocked overnight at $4^{\circ} \mathrm{C}$ in 5\% non-fat dry milk and TBS-T at pH $7.5(10 \mathrm{mM}$ Tris base, $450 \mathrm{mM} \mathrm{NaCl}$ and $0.1 \%$ Tween-20), and then were incubated with either an anti-HDAC antibody (Active Motif, Japan) or anti-iNOS polyclonal antibody (Santa Cruz Biotechnology, Inc., Santa Cruz, CA, USA). After incubation with horseradish peroxidase anti-rabbit IgG antibody (Santa Cruz Biotechnology, Inc.), the immunoreactive bands were visualized using enhanced chemiluminescence reagents (ECL Plus Western Blotting Detection System; GE Healthcare UK, Ltd.).

Bisulfite treatment. DNA was isolated from cells using a QIAamp DNA mini kit (Qiagen, Tokyo, Japan). DNA, $0.5 \mu \mathrm{g}$, was subjected to bisulfite treatment using an EZ DNA Methylation kit (Zymo Research, Orange, CA, USA), after which the bisulfite-treated DNA was stored at $-20^{\circ} \mathrm{C}$ until use.

Bisulfite DNA sequencing analysis. Bisulfite-treated DNA $1 \mu 1$, was amplified using a pair of specific primers in a total volume of $20 \mu \mathrm{l}$. For bisulfite genomic sequencing of AID, the promoter was amplified using the following primer pairs: AID1-F-5'-TTTTGTTTAGGTTTTGTTAAGTAAT-3', AID1-R-5'-TAAACAATACAACTAAAATCTCTTCTC-3', AID2-F-5'-ATGGTTTAATTTGTGTGATTTTG-3', AID2R-5'-TACATTATACGCACTACTTCTTTTAC-3', AID3-F5'-TTTTTCGTGGGTGATTGTATTGG-3' and AID3-R-5'ATAATAAACACAATCATAATAACTTTC-3'.

PCR conditions were: 35 cycles at $94^{\circ} \mathrm{C}$ for $30 \mathrm{sec}, 58^{\circ} \mathrm{C}$ for $1 \mathrm{~min}$ and $72^{\circ} \mathrm{C}$ for $1 \mathrm{~min}$. The resultant PCR product was then purified using a QIAquick PCR Purification kit (Qiagen), after which the purified PCR products were sequenced using the following primers: AID-SQ1-5'-CCCACTAAATCCCTC CCATAACAT-3', AID-SQ2-5'-CAAACCTTTAATACCAT CTATACCT-3', AID-SQ3-5'-CAAATTTACTCTAAAAAA AAAACCAATAC-3' and AID3-R-5'-ATAATAAACACAA TCATAATAACTTTC-3'.

Histone acetyltransferase (HAT) and HDAC activity assay. Nuclear protein was extracted from cells using a Nuclear Extract kit (Active Motif Japan), and HAT and HDAC activity were measured using HAT (BioVision Research Products, Mountain View, CA, USA) and HDAC assay kits (Active Motif Japan).

\section{Results}

Effect of treatment with Dnmt and HDAC inhibitors on AID expression. We examined whether or not AID expression in cancer cell lines was regulated by epigenetics. Fig. 1A describes the effects of treatment with 5-Aza-dC (5 $\mu \mathrm{M})$, a Dnmt inhibitor, on AID expression in several cancer cell lines at $10 \mathrm{~h}$ after treatment with TNF- $\alpha$. Fig. 1B shows the time course of AID expression (mRNA) after treatment with TNF- $\alpha$. AID expression was induced by additional treatment 


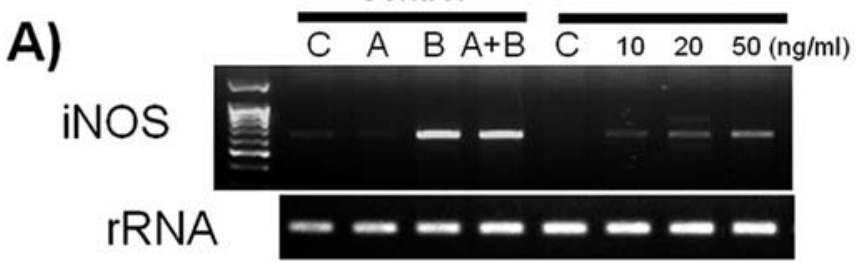

B)

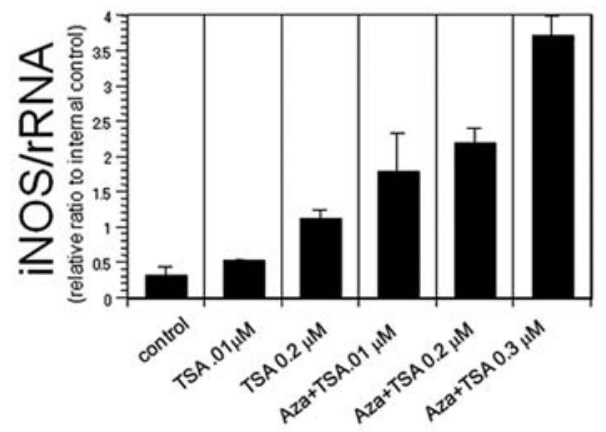

Figure 3. Effects of treatment with Dnmt and HDAC inhibitors on iNOS expression. PANC-1 cells were treated with HDAC inhibitors or TNF- $\alpha$ for $24 \mathrm{~h}$ after a 3-day treatment with 5-Aza-dC $(5 \mu \mathrm{M})$, and then iNOS mRNA expression was examined. (A) Effect of $2 \mathrm{mM}$ sodium butyrate on iNOS expression was analyzed using RT-PCR. C, control; A, 5-Aza-dC; B, sodium butyrate. (B) Effects of the HDAC inhibitor Trichostatin A on iNOS expression were examined using real-time RT-PCR. Aza, 5-Aza-dC; TSA, Trichostatin A.

with sodium butyrate, an HDAC inhibitor and GSNO, a NO donor, for 0 and $12 \mathrm{~h}$ later with TNF- $\alpha$ stimulation in LoVo and PANC-1 cell by real-time RT-PCR (Fig. 1C). AID expression was induced by additional treatment with other HDAC inhibitors, valproic acid and TSA in LoVo cells (Fig. 1D)
Methylation state at $\mathrm{Cp} G$ sites in the promoter and exon 1 regions of the AID gene. Using the bisulfite-sequence method, we were able to identify 11 methylated cytosine residues in the AID promoter and exon 1 regions (Fig. 2A). The difference in methylation status was observed in human cancer cell lines (Fig. 2B). In addition, expression of AID mRNA at $10 \mathrm{~h}$ after TNF- $\alpha$ administration was quantified by image analysis and classified into one of three categories $(-,+$ or ++$)$ and then correlated with hypomethylation status (Fig. 2B).

Effect of treatment with Dnmt and HDAC inhibitors on iNOS expression. Expression of the iNOS gene was induced using the HDAC inhibitor sodium butyrate $(2 \mathrm{mM})$ after a 3-day treatment with the Dnmt inhibitor 5-Aza-dC $(5 \mu \mathrm{M})$ in PANC-1 cells without cytokine stimulation (Fig. 3A). Observation found that HDAC inhibitor-induced iNOS expression was higher in the cells treated with 5-Aza-dC than in those treated with TNF- $\alpha$. Expression was also observed on treatment with another HDAC inhibitor, Trichostatin A (TSA), using real-time RT-PCR (Fig. 3B).

Effect of NO on AID expression and HDAC proteins and activity. Treatment with the NO donor GSNO enhanced AID expression in LoVo cells treated with 5-Aza-dC $(5 \mu \mathrm{M})$ (Fig. 4A). LoVo cells were treated with GSNO $0.5 \mathrm{mM}$ for 24 or $48 \mathrm{~h}$ and then harvested. Cytoplasm and nuclear proteins were isolated and HDAC proteins were detected by Western blotting using anti-HDAC antibody (Fig. 4B). GSNO treatment reduced cytoplasm protein levels of HDAC, but no effect of NO on nuclear protein levels of HDAC was observed. In addition, nuclear proteins had no significant effect on histone acetyltransferase activity (data not shown). However, treatment with GSNO at concentrations $0.5 \mathrm{mM}$ inhibited

A)

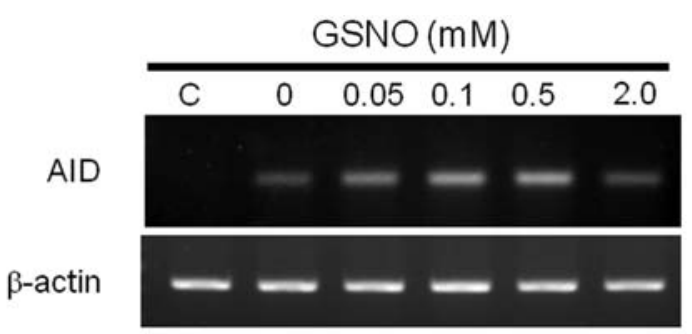

B)
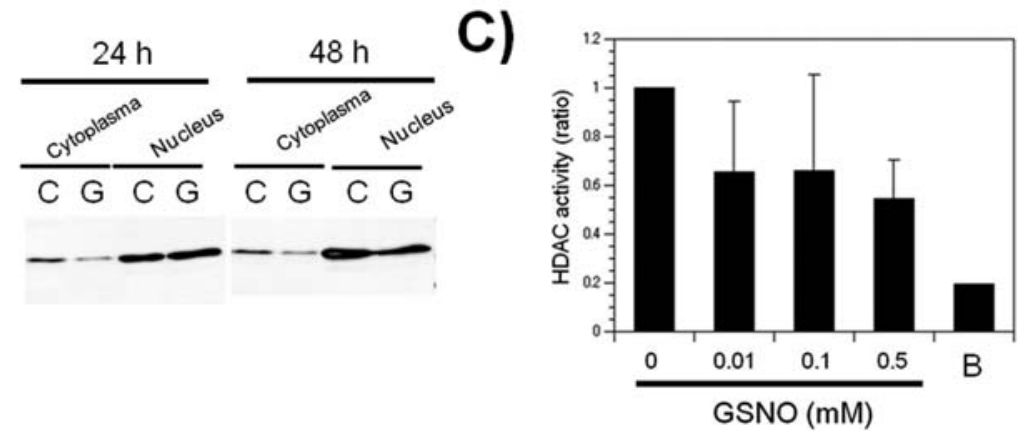

Figure 4. Effect of NO on AID expression and HDAC activity. (A) Effects of the NO donor GSNO (S-nitroso-L-glutathione) on AID expression were examined in LoVo cells after a 3-day treatment with 5-Aza-dC. (B) LoVo cells were treated with GSNO $0.5 \mathrm{mM}$ for 24 or $48 \mathrm{~h}$ and then harvested. Cytoplasm and nuclear proteins were isolated and Western blotting were perform using anti-HDAC antibody. (C) Nuclear protein was extracted from LoVo cells treated with $0.5 \mathrm{mM}$ of GSNO for $24 \mathrm{~h}$, and then the HDAC activity of the nuclear protein was measured using an HDAC assay kit. C, control; G, GSNO; B, sodium butyrate. 
A)

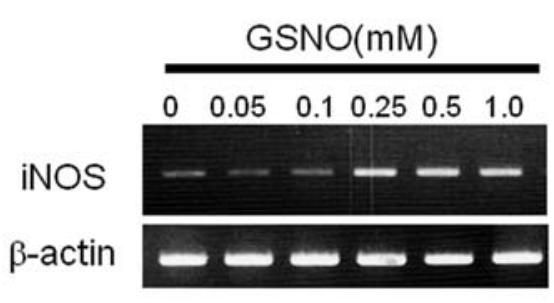

B)

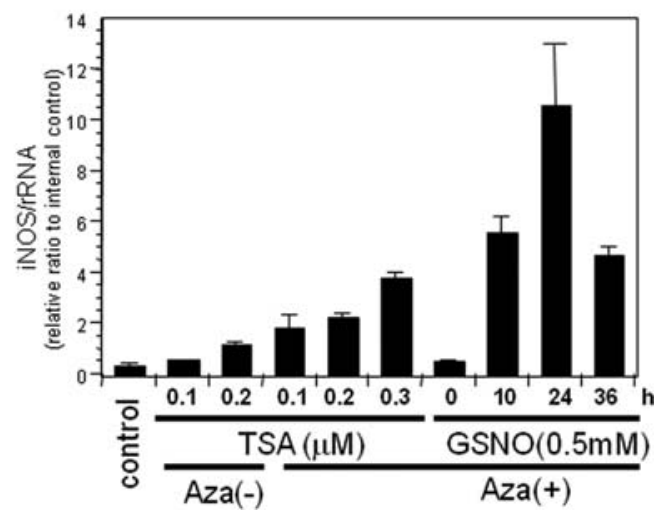

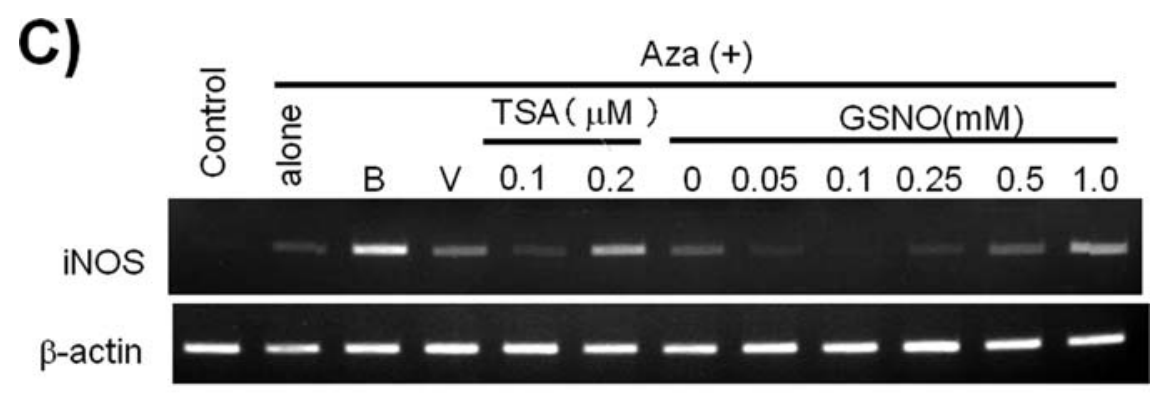

D)

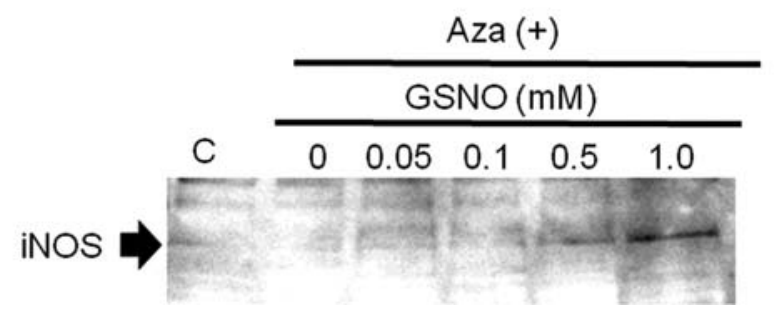

Figure 5. Effect of NO on expression of the AID and iNOS genes in cancer cell lines. Effects of the NO donor GSNO and HDAC inhibitor TSA on iNOS expression were examined in LoVo cells (A) and (B) after 3-day treatment with 5-Aza-dC (5 $\mu \mathrm{M})$. On further examination using SW480, another colon cancer cell line, HDAC inhibitors [sodium butyrate $(2 \mathrm{mM})$, valproic acid (5 mM) and TSA] and GSNO (S-nitroso-L-glutathione) enhanced iNOS expression in the SW480 cells treated with 5-Aza-dC (5 $\mu \mathrm{M})$ [RT-PCR (C) and Western blotting (D)]. B, sodium butyrate; V, valproic acid; TSA, Trichostatin A.

HDAC activity in LoVo cells (by $~ 50 \%$ ) (Fig. 4B). NOinduced HDAC inhibition peaked at $24 \mathrm{~h}$ after GSNO treatment and continued for three days (data not shown).

Effects of NO on iNOS expression. We examined iNOS expression levels using RT-PCR and real-time PCR in LoVo cells by administering GSNO or TSA after a 3-day treatment with 5-Aza-dC (5 $\mu \mathrm{M})$ (Fig. 5A and B). On further examination using SW480, another cancer cell line, HDAC inhibitors (sodium butyrate, valproic acid and TSA) and GSNO enhanced iNOS expression in the SW480 cells treated with 5-Aza-dC (5 $\mu \mathrm{M})$ (Fig. 5C and D; RT-PCR and Western blotting). SNAP, another NO donor, also enhanced iNOS expression (data not shown).

\section{Discussion}

Here, we examined whether or not deregulation of epigenetics is involved in the ectopic expression of AID, and whether or not NO modulates this expression. Our findings suggested that epigenetic deregulation is indeed involved in the ectopic AID expression, and that NO is able to enhance AID expression in cancer cells. Further, we observed that NO is capable of modulating HDAC activity.

Large amounts of NO are capable of being produced by iNOS during inflammatory processes, which can subsequently induce pathophysiological changes in carcinogenic processes (18). Recently, research has been focused on aberrant hypermethylation in tumor suppressor genes, such as p16 (15). In a previous study, we used p53-iNOS double mutant mouse embryofibroblast (MEF) cells to examine whether or not NO induced de novo hypermethylation on the $\mathrm{CpG}$ island in the promoter region of $\mathrm{p} 16$. However, while hypermethylation was not observed at all in iNOS ${ }^{+/+}$MEF cells, it was frequently observed in MEF cells without the iNOS gene (19). These observations suggest that NO may inhibit methylation.

Physiologically, AID expression is restricted to activated B cells (2). DNA methylation and histone modification are important epigenetic mechanisms in cooperatively controlling cell-specific gene expression, and Dnmt and HDAC activities 
therefore play a critical role in maintaining epigenetic regulation. In the present study, inhibitors of Dnmt and HDAC enhanced AID expression, and all CpG sites in the promoter region were methylated in the genome extracted from normal human blood cells (unpublished data). However, a number of cancer cell lines were observed to be hypomethylated, and this hypomethylation status was found to be correlated with AID expression, suggesting that AID expression may be regulated by an epigenetic-based mechanism.

Chan et al (20) reported that iNOS gene expression is regulated by epigenetic-based gene silencing in vascular endothelial cells, and our present results are consistent with these previous findings. We further noted that administration of NO donors induced iNOS expression in cancer cells treated with 5-Aza-dC, with no stimulation from factors such as TNF- $\alpha$. The precise mechanisms regarding how NO enhances AID and iNOS expression and how NO affects HDAC activity remain unknown. We believe that NO enhanced AID and iNOS expression in Lovo cells by inhibiting HDAC activity. However, the effects of NO on HDAC activity differ between cell lines. For example, NO reduced HDAC activity in LoVo and PANC-1 cells, but increased HDAC activity in DLD-1 cells, all without affecting protein levels of HDACs (unpublished data). Regarding these points, further studies are needed to examine the effects of $\mathrm{NO}$ on HDAC activity.

Several cytokines, chemokines, reactive nitrogen and oxygen species, and several types of prostaglandins are released during inflammation-associated carcinogenesis, all of which can induce AID expression, an important mechanism of inflammatory-associated carcinogenesis (21). Recent studies have noted that aberrant AID expression elevates the p53 mutation rate (6). Functional loss of p53 can induce iNOS overexpression, thereby leading to production of a large amount of NO $(13,22)$. In addition, methylated $\mathrm{CpG}$ at specific sites has been found to be demethylated by longterm exposure with inflammatory cytokines (23).

Our preliminary data suggest that NO could enhance AID expression in cancer cells with a demethylated $\mathrm{CpG}$ in exon 1-promotor region, resulting in greater frequencies of gene mutation. The vicious cycle of NO in the inflammatory process (iNOS to AID to p53 mutation to iNOS) may be involved in inflammatory-associated carcinogenesis. Further, given recent plans to introduce Dnmt and HDAC inhibitors as novel cancer treatments, these findings that Dnmt and HDAC inhibitors may enhance expression of AID and iNOS, resulting in further cancer progression, might be taken into consideration.

\section{Acknowledgements}

This study was supported in part by the Grant-in-Aid for Cancer Research (16S-1 and 19S-1) from the Ministry of Health and Welfare of Japan.

\section{References}

1. Muramatsu M, Kinoshita K, Fagarasan S, Yamada S, Shinkai Y and Honjo T: Class switch recombination and hypermutation require activation-induced cytidine deaminase (AID), a potential RNA editing enzyme. Cell 102: 553-563, 2000.
2. Muto T, Muramatsu M, Taniwaki M, Kinoshita K and Honjo T: Isolation, tissue distribution, and chromosomal localization of the human activation-induced cytidine deaminase (AID) gene. Genomics 68: 85-88, 2000.

3. Komori J, Marusawa H, Machimoto T, et al: Activation-induced cytidine deaminase links bile duct inflammation to human cholangiocarcinoma. Hepatology 47: 888-896, 2008.

4. Endo Y, Marusawa H, Kinoshita K, et al: Expression of activation-induced cytidine deaminase in human hepatocytes via NF-kappaB signaling. Oncogene 26: 5587-5595, 2007.

5. Matsumoto Y, Marusawa H, Kinoshita K, et al: Helicobacter pylori infection triggers aberrant expression of activation-induced cytidine deaminase in gastric epithelium. Nat Med 13: 470-476, 2007.

6. Takai A, Toyoshima T, Uemura M, et al: A novel mouse model of hepatocarcinogenesis triggered by AID causing deleterious p53 mutations. Oncogene 28: 469-478, 2009

7. Forstermann $U$ and Kleinert H: Nitric oxide synthase: expression and expressional control of the three isoforms. Naunyn Schmiedebergs Arch Pharmacol 352: 351-364, 1995.

8. Lala PK and Chakraborty C: Role of nitric oxide in carcinogenesis and tumour progression. Lancet Oncol 2: 149-156, 2001.

9. Ogura T, DeGeorge G, Tatemichi M and Esumi H: Suppression of anti-microtubule agent-induced apoptosis by nitric oxide: possible mechanism of a new drug resistance. Jpn J Cancer Res 89: 199-205, 1998.

10. Fukunaga-Takenaka R, Fukunaga K, Tatemichi M and Ohshima H: Nitric oxide prevents UV-induced phosphorylation of the p53 tumor-suppressor protein at serine 46: a possible role in inhibition of apoptosis. Biochem Biophys Res Commun 308: 966-974, 2003.

11. Chin K, Kurashima Y, Ogura T, Tajiri H, Yoshida S and Esumi H: Induction of vascular endothelial growth factor by nitric oxide in human glioblastoma and hepatocellular carcinoma cells. Oncogene 15: 437-442, 1997.

12. Ishii Y, Ogura T, Tatemichi M, Fujisawa H, Otsuka F and Esumi H: Induction of matrix metalloproteinase gene transcription by nitric oxide and mechanisms of MMP-1 gene induction in human melanoma cell lines. Int J Cancer 103: $161-168,2003$.

13. Tatemichi M, Tazawa H, Masuda M, et al: Suppression of thymic lymphomas and increased non-thymic lymphomagenesis in Trp53-deficient mice lacking inducible nitric oxide synthase gene. Int J Cancer 111: 819-828, 2004.

14. Tatemichi M, Ogura $\mathrm{T}$ and Esumi $\mathrm{H}$ : Impact of inducible nitric oxide synthase gene on tumor progression. Eur J Cancer Prev 18: 1-8, 2009.

15. Wink DA, Kapspzak KS, Maragos CM, et al: DNA deaminating ability and genotoxicity of nitric oxide and its progenitors. Science 254: 1001-1003, 1991.

16. Ushijima T and Okochi-Takada E: Aberrant methylations in cancer cells: where do they come from? Cancer Sci 96: 206-211, 2005.

17. Sharma S, Kelly TK and Jones PA: Epigenetics in cancer. Carcinogenesis 31: 27-36, 2010.

18. Ohshima H, Tatemichi $M$ and Sawa T: Chemical basis of inflammation-induced carcinogenesis. Arch Biochem Biophys 417: 3-11, 2003.

19. Tatemichi M, Hata H, Tazawa $H$ and Nakadate T: Lipopolysaccharide induces aberrant hypermethylation of Hic-1 in mouse embryonic fibroblasts lacking p53 gene. Anticancer Res 28: 2101-2108, 2008

20. Chan GC, Fish JE, Mawji IA, Leung DD, Rachlis AC and Marsden PA: Epigenetic basis for the transcriptional hyporesponsiveness of the human inducible nitric oxide synthase gene in vascular endothelial cells. J Immunol 175: 3846-3861, 2005.

21. Chiba T and Marusawa H: A novel mechanism for inflammationassociated carcinogenesis; an important role of activationinduced cytidine deaminase (AID) in mutation induction. J Mol Med 87: 1023-1027, 2009.

22. Ambs S, Ogunfusika MO, Merriam WG, Bennett WP, Billiar TR and Harris CC: Up-regulation of inducible nitric oxide synthase expression in cancer-prone p53 knockout mice. Proc Natl Acad Sci USA 95: 8823-8828, 1998.

23. Hashimoto K, Oreffo, Gibson, et al: DNA demethylation at specific $\mathrm{CpG}$ in the IL1B promoter in response to inflammatory cytokines in human articular chonrocytes. Arthritis Rheum 60: 3303-3313, 2009. 